\title{
Zur gesellschaftlichen Wahrnehmung des voll autonomen Fahrens und seiner möglichen nachhaltigkeits- bezogenen Implikationen
}

Ergebnisse einer Repräsentativbefragung

\begin{abstract}
Jürgen Hampel*, Universität Stuttgart, Institut für Sozialwissenschaften, Abteilung für Technik- und Umweltsoziologie, Seidenstraße 36, 70174 Stuttgart (Juergen.Hampel@sowi.uni-stuttgart.de)

Cordula Kropp, Universität Stuttgart, Institut für Sozialwissenschaften, Abteilung für Technik- und Umweltsoziologie, Seidenstraße 36, 70174 Stuttgart (cordula.kropp@sowi.uni-stuttgart.de)

Michael M.Zwick, Universität Stuttgart, Institut für Sozialwissenschaften, Abteilung für Technik- und Umweltsoziologie, Seidenstraße 36, 70174 Stuttgart (michael.zwick@sowi.uni-stuttgart.de)

* Die Autorlnnen sind in alphabetischer Folge aufgelistet.
\end{abstract}

Dieser Artikel thematisiert die Nachhaltigkeit des autonomen Fahrens. Die Daten des TechnikRadars, einer für die deutsche Wohnbevölkerung repräsentativen Bevölkerungsumfrage, aktuell zur Digitalisierung, erlauben es, methodisch belastbar Aussagen über die Wahrnehmung des autonomen Fahrens zu machen. Der Artikel fügt dem bislang vorrangig expertenbasierten Diskurs explizit die Stimme der Öffentlichkeit hinzu. Obwohl das System des autonomen Fahrens noch nicht hinreichend definiert ist, können für die weitere Entwicklung bedeutsame Aspekte aufgezeigt werden: Autonomes Fahren trifft derzeit nicht auf einen gesellschaftlichen Bedarf, die Delegation des Autofahrens an technische Systeme wird nur von einer Minderheit begrüßt. Skepsis finden wir vor allem bei der sozialen Nachhaltigkeit autonomer Mobilität; insbesondere werden Sicherheitsmängel und die Anfälligkeit des Systems, etwa gegenüber Angriffen von Cyber-Kriminellen, befürchtet.

\section{The social perception of fully autonomous driving and its possible sustainability-related implications \\ Results of a representative survey in Germany}

This article discusses the sustainability of autonomous driving. The empirical analyses presented in this article are based on data of the first

This is an article distributed under the terms of the Creative Commons Attribution License CCBY 4.0 (https://creativecommons.org/licenses/by/4.0/)

https://doi.org/10.14512/tatup.27.2.38

Submitted: 30.04 .2018 . Peer reviewed. Accepted: 07.06.2018
TechnikRadar, a survey on the perception of technology with representative for the German population. While most of the literature on autonomous driving focuses on expert views on this issue, this paper adds the perspective of the general public. Although autonomous driving as a socio-technical system is far from being fully developed, making it impossible to assess the ecological effects of its applications, the TechnikRadar allows studying the perceived social sustainability of autonomous driving. At the moment, public perception of autonomous driving is dominated by fears regarding safety, the protection of data produced by the system, and the system's vulnerability, in particular to attacks by cyber criminals.

Keywords: autonomous driving, sustainability, public perception

\section{Einleitung}

$\mathrm{Zu}$ den gegenwärtig intensiv diskutierten Themen, sowohl in der politischen Arena als auch in der Wissenschaft, gehört die Zukunft der Mobilität. Dazu haben eine Vielzahl von Entwicklungen beigetragen, die nicht zuletzt Folgen der massiven Verbreitung individueller Mobilität sind. Grunwald und Kopfmüller (2012, S. 117) nennen beispielhaft ,Treibhausgasemissionen, umwelt- und gesundheitsschädliche Schadstoffemissionen $\left(\mathrm{NO}_{\mathrm{x}}\right.$, 
Feinstaub usw.), Lärmbelastungen, Ressourcenverbrauch sowie Flächenversiegelung, Landschaftszerschneidung und hieraus resultierende Biodiversitätsverluste“.

Während das gegenwärtig dominante Mobilitätsmodell in das Zentrum gesellschaftlicher Auseinandersetzungen gerät, stehen neue technische Entwicklungen vor der Tür, von denen angenommen wird, dass sie Mobilität neu definieren. Zu diesen Neuerungen gehört das autonome Fahren, ein Konzept, bei dem Menschen nicht mehr als (alleinige) Fahrzeuglenker in Erscheinung treten, sondern die Kontrolle und Steuerung des Verkehrs durch (auch) vernetzte Computersysteme erfolgt. Noch steckt das autonome Fahren in den Kinderschuhen; gegenwärtig steht in erster Linie die technische Funktionsfähigkeit autonomer Fahrzeuge auf dem Prüfstand. Wie autonomes Fahren als Verkehrssystem organisiert werden wird, ist dagegen noch nicht absehbar, viele Möglichkeiten sind denkbar. Eine Abschätzung der Folgen autonomer Mobilität ist daher zum gegenwärtigen Zeitpunkt kaum sicher möglich. Gerade deshalb ist eine Diskussion über die Gestaltung des autonomen Verfahrens von erheblicher Bedeutung, als es darum geht, Parameter festzulegen, an denen sich das System autonomes Fahren orientieren soll.

Gegenwärtig wird dieser Diskurs vor allem als Expertendiskurs geführt. Das TechnikRadar, eine von ZIRIUS an der Universität Stuttgart durchgeführte und von acatech, der deutschen Akademie für Technikwissenschaften, und der Körber-Stiftung geförderte repräsentative Untersuchung der Einstellungen der deutschen Öffentlichkeit zum Thema Technik, die sich in ihrer 2018er Studie schwerpunktmäßig mit Fragen der Digitalisierung befasst hat, erlaubt es, diesen Expertendiskurs um die Perspektive der Öffentlichkeit zu ergänzen.

\section{Nachhaltige Mobilität}

Die Diskussion um die Zukunft der Mobilität findet dominant unter dem Label „Nachhaltige Mobilität“" statt (etwa Umweltbundesamt 2005), womit der allgemeine Nachhaltigkeitsdiskurs mit dem Mobilitätsdiskurs verbunden wird. Zahlreiche Projekte, von technischen Entwicklungsprojekten bis hin zu Reallaboren, die versuchen, alternative Mobilitätskonzepte zu initiieren, zielen darauf ab, Mobilität und das Mobilitätssystem in Richtung Nachhaltigkeit zu verändern.

So verbreitet Nachhaltigkeit als Prinzip ist, so schwer fällt es bis heute, Nachhaltigkeit präzise zu definieren. Auch wenn zahlreiche Versuche unternommen wurden, wissenschaftlich nachvollziehbare Nachhaltigkeitskonzepte zu entwickeln, so ist der Begriff Nachhaltigkeit nach wie vor alles andere als eindeutig bestimmt. Konkurrierende Vorstellungen darüber, was Nachhaltigkeit ist, sind auch in Zukunft zu erwarten, was nicht zuletzt daran liegt, dass Nachhaltigkeit weniger ein wissenschaftliches Prinzip als ein ethischer Imperativ ist (Grunwald und Kopfmüller 2012, S. 11). Die Brundlandt-Kommission hat Nachhaltigkeit als ein Prinzip formuliert, das die Bedürfnisse der gegenwärtigen Generation befriedigt, ohne die Möglichkeit künftiger
Generationen zu beeinträchtigen, ihre Bedürfnisse zu befriedigen (Hauff 1987). Dieses Nachhaltigkeitskonzept ist anthropozentrisch, es stellt den Menschen, genauer seine Bedürfnisse, in den Mittelpunkt. Die ökologische Frage stellt sich hier nur insofern, als eine nicht-nachhaltige Entwicklung die Möglichkeiten künftiger Generationen, ihre Bedürfnisse zu befriedigen, in Frage stellt. In der Rio-Erklärung von 1992 (Vereinte Nationen 1992) wurde die gesellschaftliche Dimension von Nachhaltigkeit noch einmal verstärkt, indem Prinzipien wie die Bekämpfung der Armut oder die Beteiligung aller betroffenen Bürgerinnen und Bürgern eingefordert wurden.

\section{Die Ergebnisse des TechnikRadars ergänzen den bisherigen Experten- diskurs zum autonomen Fahren um die Perspektive der Öffentlichkeit.}

In der Nachhaltigkeitsforschung sind Modelle verbreitet, die mehrere Nachhaltigkeitsdimensionen unterscheiden, am häufigsten zwischen ökologischer, sozialer und ökonomischer Nachhaltigkeit als Bewertungsdimensionen, zwischen denen durchaus konfliktäre Beziehungen möglich sind. Im HGF-Konzept (Kopfmüller et al. 2001) gehört die Bewahrung der Entwicklungs- und Handlungsmöglichkeiten genauso zur Nachhaltigkeit wie die Erhaltung des gesellschaftlichen Produktivpotenzials und die Sicherung der menschlichen Existenz.

Renn et al. 2007 gehen von drei normativ gesetzten Nachhaltigkeitsfunktionen aus (2007, S. 41), nämlich der Sicherstellung gesellschaftlicher, ökonomischer und ökologischer Funktionen für kommende Generationen (systemare Integrität), der Durchsetzung von Gerechtigkeitsnormen innerhalb und zwischen Generationen und dem dauerhaften Erhalt der individuellen Lebensqualität, wenn auch die Unterscheidung bei der Festlegung der Indikatoren nicht aufrechterhalten wird. So wird der Anteil des öffentlichen Personenverkehrs an der Personenbeförderung als Indikator für Lebensqualität definiert (2007, S. 77).

Grunwald und Kopfmüller (2012, S. 117) sehen Mobilität als wesentliches gesellschaftliches Bedürfnis. Mobilität sei einerseits Voraussetzung für Teilhabe, andererseits Voraussetzung für Beschäftigung und Wohlstand. Sie definieren Nachhaltigkeit im Verkehrsbereich, sich auf andere Studien beziehend, als „Zugang möglichst aller Menschen zu sicheren Verkehrsdienstleistungen, die ihnen die Teilhabe am gesellschaftlichen Leben ermöglichen, die erschwinglich sind und die möglichst geringe Umwelt- und Gesundheitsbelastungen bewirken" (Grunwald und Kopfmüller 2012, S. 119 f.). Diese Definition, die wir für diese Arbeit übernehmen, unterscheidet die folgenden Bewertungsdimensionen für nachhaltige Mobilität: Umwelt- und Gesundheitsbelastungen, Teilhabemöglichkeiten, Kosten und Sicherheit. 
In Anbetracht des gegenwärtigen Entwicklungsstands autonomer Mobilität ist es noch nicht möglich, hinreichend präzise die Nachhaltigkeit dieser Innovation und vor allem der mit ihr verbundenen Nutzungen und Anwendungen zu analysieren. Die für die Auswirkungen entscheidendere Frage, wie autonomes Fahren als sozio-technisches System konfiguriert wird, ist noch nicht einmal in Ansätzen geklärt (vgl. auch Schreurs und Steuwer 2015, S. 161; Dangschat 2017, S. 496, Thomopoulos und Givoni 2015, S.9). Wird sich eine bloße technische Aufrüstung des jetzigen Individualverkehrs durchsetzen? Werden sich Callby-demand-Systeme durchsetzen, bei denen sich Nutzer ein Gefährt wie ein Taxi für einen bestimmten Fahrtweg mieten kön- den können, wäre durch autonome Mobilität eher noch eine $\mathrm{Zu}$ nahme des Individualverkehrs (Thomopoulos und Givoni 2015, S. 9) und ein Beitrag zu einer weiteren Zersiedelung der Landschaft (Dangschat 2017, S. 504) zu erwarten.

Auch wenn hinsichtlich der Gestaltung von autonomem Fahren noch zahlreiche Entscheidungen ausstehen, ist es an der Zeit, dem bislang eher von Experten getragenen Diskurs - seien es Technikexperten, seien es Experten aus dem Gebiet der Technikfolgenabschätzung - die Perspektive der Öffentlichkeit gegenüberzustellen. Wir wollen in unserem Beitrag einer bedürfnisorientierten Nachhaltigkeitskonzeption, wie sie von der Brundlandt-Kommission vorgeschlagen wird, und der Definition

\section{Es ist an der Zeit, dem bislang eher von Experten getragenen Diskurs über autonomes Fahren die Perspektive der Öffentlichkeit gegenüberzustellen.}

nen (ride sharing)? Oder sind andere Formen zu erwarten, etwa in der Art von Sammeltaxen, die nachfragebedingt optimierte Routen bedienen? Oder wird es lediglich eine gleichmäßige Geschwindigkeitssteuerung aller Fahrzeuge geben, um die Kapazität des Straßensystems optimieren zu können? Je nach Gestaltung des autonomen Fahrens als System werden die Auswirkungen in ökologischer, ökonomischer und sozialer Hinsicht unterschiedlich aussehen.

Vor diesem Hintergrund ist es nicht überraschend, dass auch in der Literatur die Auswirkungen des autonomen Fahrens als ungewiss eingeschätzt werden (Wadud et al. 2016, S. 16). Autonomes Fahren wird als Thema der industriellen Wettbewerbsfähigkeit, der Ressourcen-Effizienz und der Nachhaltigkeit gesehen (Schreurs und Steuwer 2015, S. 155). Dabei kommen die meisten Studien zu optimistischen Ergebnissen, was nicht zuletzt daran liegt, dass sie sich mit Fragen der technischen Machbarkeit beschäftigen. Die Literatur zum autonomen Fahren diskutiert die Auswirkungen dieser Innovation daher vorrangig auf der Grundlage von Erwartungen und Visionen, ist also eher Vision Assessment als Technology Assessment im engeren Sinn. Dabei gibt es nicht nur positive, sondern auch negative Utopien des autonomen Fahrens.

Positive Erwartungen beziehen sich auf die Effizienzgewinne bei der Mobilität (u. a. Funk 2015, S. 867; Friedrich 2015, S. 312), aber auch auf die Aussicht, die Teilhabemöglichkeiten von mobilitätseingeschränkten Menschen zu erhöhen (Grunwald 2015, S. 668; Dangschat 2017, S. 497). Zu den befürchteten negativen Auswirkungen zählt die Zunahme der Mobilität gerade dadurch, dass Mobilität bequemer wird und autonome Mobilität auch in einem Konkurrenzverhältnis zu öffentlichen Verkehrsmitteln und zu nicht-motorisierten Formen individuellen Personennahverkehrs steht (Dangschat 2017; S. 497 f.). Bedenkt man zudem, dass Pendelzeiten für andere Tätigkeiten genutzt wer- nachhaltiger Mobilität von Grunwald und Kopfmüller (2012) folgen, und uns der Frage zuwenden, ob und wie autonomes Fahren nach Einschätzung der Öffentlichkeit zu einer nachhaltigen Mobilität beitragen kann.

Da die ökologischen Auswirkungen des voll autonomen Fahrens noch nicht abgeschätzt werden können, steht in diesem Beitrag die soziale Dimension der Nachhaltigkeit im Mittelpunkt, insbesondere die Frage nach der Ermöglichung von Teilhabe ansonsten ausgeschlossener Menschen und die Frage nach der Sicherheit der Mobilität, wozu die Sicherheit vor Unfällen gehört, aber auch, auf einer abstrakteren Ebene, die Sicherheit des Verkehrssystems autonomer Mobilitität.

\section{Der Datensatz}

Die Frage, wie die deutsche Öffentlichkeit zum voll autonomen Fahren steht, welche Erwartungen und Bedenken sie an diese Technologie heranträgt, unter welchen Bedingungen ihre Nutzung in Frage käme, werden wir anhand der aktuellen TechnikRadar-Befragung diskutieren. Beim TechnikRadar handelt es sich um eine turnusmäßige Repräsentativbefragung der über 16-jährigen, deutschsprachigen Wohnbevölkerung, die Haltungen der Öffentlichkeit gegenüber zukunftsweisenden Technologien zum Gegenstand hat. Die Studie reagiert - sieht man von einigen wenigen Eurobarometer-Befragungen $\mathrm{ab}$ - auf den seit der Jahrtausendwende anhaltenden Mangel an empirischer Technikberichterstattung (Hampel und Zwick 2016, S. 28).

Die erste Welle des Technik-Radars ist dem Thema Digitalisierung und Automatisierung gewidmet, mit den Schwerpunkten Smart Home, Pflegeroboter und Autonomes Fahren. Für autonomes Fahren werden in der Literatur vier Entwicklungsstadien diskutiert, die sich vor allem in Bezug auf die Kontrolle durch 


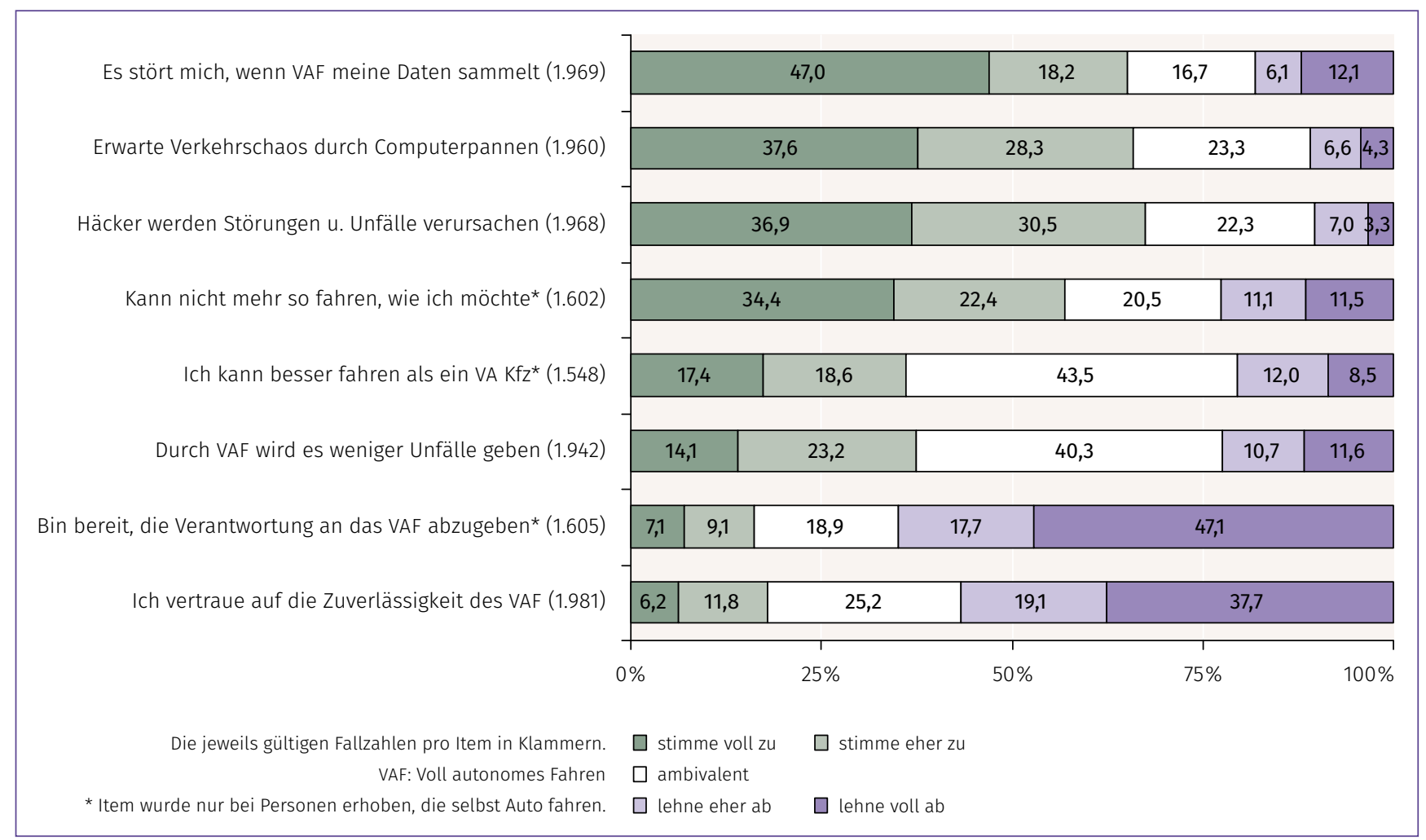

Abb.1: Die Einstellung der Öffentlichkeit zum vollautonomen Fahren.

Quelle: Eigene Darstellung auf Basis der Technik-Radar-Befragung 2017

Fahrzeuglenker unterscheiden": Stufe 1 bezeichnet das assistierte Fahren, etwa durch Antiblockiersysteme oder Spurhalteassistenten. Stufe 2 meint Teilautomatisierung, worunter beispielsweise Einparkhilfen oder so genannte Stauassistenten fallen. Entwicklungen dieser beiden Stufen werden bereits eingesetzt. Stufe 3 steht für hoch automatisiertes Fahren, worunter das selbständige Fahren auf der Autobahn oder im Stau fällt und die Umweltbeobachtung von den Fahrzeugtechnologien vollständig übernommen wird. Das vollautomatische Fahren im Stadtverkehr oder die fahrerlose Parkplatzsuche gipfeln in Stufe 4. Sie werden als vollautomatisiertes Fahren klassifiziert (Verband der Automobilindustrie 2015, S. 15), das nach den Ergebnissen einer Studie des Fraunhofer-Instituts für Arbeitswirtschaft und Organisation (IAO) in Deutschland vermutlich nicht vor 2030 die Marktreife erreichen wird (Cacilo et al. 2015, S. 9). Die thematische Breite und die begrenzte Befragungsdauer erzwangen allerdings Selektionen. Mit Blick auf die gewünschte zukunftsweisende Perspektive der Befragung fand deshalb ausschließlich das vollautonome Fahren Eingang in das TechnikRadar.

Die telefonische Befragung (CATI) wurde im Oktober und November 2017 von der INFO GmbH Markt- und Meinungs-

1 Die im TechnikRadar verwendete Klassifizierung von Stufen des autonomen Fahrens unterscheidet sich von anderen Klassifizierungssystemen, auf die in diesem TATuP-Thema verwiesen wird. Siehe dazu die Einleitung von Torsten Fleischer und Jens Schippl. forschung auf Basis einer Zufallsstichprobe im Dual-frameModus durchgeführt. Die Stichprobengröße beträgt 2.002 Fälle. Die Ausschöpfungsquote lag bei $15 \%$, ein Wert, den Gabler und andere nach einer Analyse von Telefonbefragungen als durchschnittlich bezeichnen (2012, S. 165): gegenwärtig seien Ausschöpfungsquoten zwischen $10 \%$ und $20 \%$ normal.

\section{Das voll autonome Fahren im Urteil der deutschen Öffentlichkeit}

Natürlich wäre es vermessen anzunehmen, mit einer Stichprobe aus dem Jahr 2017 eine Prognose über die gesellschaftliche Akzeptanz des voll autonomen Fahrens in ein oder zwei Dekaden abgeben zu können. Zu groß sind die Unwägbarkeiten über Art, Leistungsfähigkeit, Zuverlässigkeit und Kosten der Technik. Wie sich autonomes Fahren entwickeln wird, hängt wesentlich von der staatlichen Regulierung beispielsweise hinsichtlich Datenschutz, Datensicherheit oder Haftungsfragen ab, sowie von der Frage, über welche Geschäftsmodelle und für welche Klientel autonom steuernde Fahrzeuge vorrangig eingeführt werden für Personenverkehr, in der Logistik, über Sharing- bzw. Mobility-on-demand-Konzepte oder als Kraftfahrzeuge in Privatbesitz? Von der Beantwortung dieser Fragen hängen nicht nur der Markterfolg und die politische Akzeptanz des voll autonomen Fahrens maßgeblich ab, sondern auch die denkbaren Implika- 
tionen für die soziale, ökonomische und ökologische Nachhaltigkeit dieser Technik.

Die Versprechen der Produzenten klingen vollmundig: Neben der möglichen Abnahme der Zahl an Fahrzeugen durch voll autonome Fahrzeuge in Verbindung mit Sharing-Konzepten und der Lösung der innerstädtischen Parkplatzprobleme mittels Sammelgaragen und Valet-Parken versprechen die Produzenten auch, dass Staus durch eine erhöhte Verkehrsdichte mit gleichmäßigerer Geschwindigkeit vermieden werden können, dass vernetzte Fahrzeuge in engerem Abstand fahren und höhere Kapazitäten von Verkehrswegen ermöglichen. Vor allem aber mit zwei Hauptargumenten wird geworben: mit verbesserter Mobilität für Personen, die aus welchen Gründen auch immer, keine Fahrerlaubnis besitzen und mit sinkenden Unfallzahlen (exemplarisch Minx und Dietrich 2015; Heymann und Meister 2017, S. 7 f.; Fraedrich et al. 2017, S. 20 ff.).

Wie aber denkt die Öffentlichkeit hierzulande über das voll autonome Fahren? Abbildung1 zeigt, dass die Bevölkerung den Versprechungen der Hersteller zum gegenwärtigen Zeitpunkt wenig Glauben schenkt. Schon vor den beiden jüngsten, tödlich verlaufenen Unfällen mit voll autonom fahrenden Kraftfahrzeugen $^{2}$ äußerten gerade einmal 16,2\% der Befragten die Bereitschaft, ihre Verantwortung an voll autonom fahrende Fahrzeuge abzugeben. Es darf vermutet werden, dass dieses Maß der Akzeptanz nach den Ereignissen im März 2018 in der Öffentlichkeit noch weiter gesunken ist. Für diese These spricht auch, dass im TechnikRadar nur 18,0\% der Befragten auf die Zuverlässigkeit voll autonom fahrender Kraftfahrzeuge vertrauen und nur eine Minderheit von 37,3\% daran glauben mochte, dass es durch voll autonome Fahrzeuge weniger Unfälle geben wird. ${ }^{3}$

Das für die soziale Nachhaltigkeit erste relevante Kriterium, nämlich die in Aussicht gestellte Absenkung der Unfallzahlen, findet in der Öffentlichkeit nur geringe Glaubwürdigkeit. Statt-

2 Vor der Befragung lag nur der fatale Unfall eines selbstfahrenden Tesla S in Florida am 7. Mai 2016 (Spiegel Online 2016), wohingegen sich die beiden jüngsten tödlichen Unfälle mit selbstfahrenden Autos am 19. März 2018 in Arizona (Spiegel Online 2018 a) und am 23. März 2018 in Kalifornien nach der Erhebung des TechnikRadars ereigneten (Spiegel Online 2018 b).

3 Diese Zahlen liegen in der Größenordnung anderer aktueller Studien zum autonomen Fahren, die jedoch auf anderen Stichprobendesigns und unterschiedlichen Itemformulierungen basieren: In der für Autofahrer in Deutschland repräsentativen ARAL-Studie vom März 2017 konnten sich $20 \%$ der Befragten vorstellen, autonom zu fahren (in der Befragungswelle von 2015 waren es noch $34 \%$ gewesen); unter den Befragten mit kritischer Haltung dominierten Sicherheitsbedenken und eine hohe Unfallgefahr (ARAL AG 2017, S. 22). Der Deloitte Konsumenten-Studie zufolge präferierten 2016 in Deutschland rund $25 \%$ der Befragten voll autonomes Fahren; $72 \%$ der Befragten zweifeln an der Sicherheit der Fahrzeuge (Deloitte 2017, S. 4 ff.). In einer Repräsentativbefragung der erwachsenen Wohnbevölkerung in Deutschland zum autonomen Fahren im Auftrag von Ernst \& Young bekundeten 2017 26\% der Befragten, sich vorstellen zu können, zukünftig mit einem autonom fahrenden Fahrzeug unterwegs zu sein; $67 \%$ sahen in der mangelnden Sicherheit dieser Fahrzeuge ein grundsätzliches Problem - in der Befragungswelle 2013 waren es nur 44\% gewesen (Fuß 2017, S. 4 und S. 8). Auch in einer für die über 14-jährige Wohnbevölkerung repräsentativen Studie der Bertelsmann-Stiftung aus dem Jahr 2017 dominierten Angst vor Unfällen bzw. fehlerhafte Technik die Einwände gegen das autonome Fahren (Bertelsmann Stiftung 2017, S. 4). dessen werden weitere Einwände gegen das voll autonome Fahren vorgebracht, die auf eine Bedrohung von Freiheits- oder gar Grundrechten hinauslaufen, wie beispielsweise das Ende der von einem Premiumhersteller intensiv beworbenen „Freude am Fahren“" ${ }^{\prime 4}$ das von $56,8 \%$ der Befragten befürchtet wird, oder aber knapp zwei Drittel der Befragten (65,2\%), die sich daran stören, wenn voll autonom fahrende Fahrzeuge personenbezogene Daten sammeln. Ähnlich häufig werden Bedenken hinsichtlich der Systemsicherheit laut. Jeweils über $65 \%$ der Befragten erwarten, dass es zu Unfällen, Störungen oder gar einem Verkehrschaos kommen wird, wenn Computerpannen auftreten oder das System von Internetkriminellen gehackt wird. Lediglich bei der Frage, ob man selber besser fahren könne als der Computer, zeichnet sich ein uneinheitliches, ambivalentes Bild ab.

Insgesamt weisen die ausgeprägten Bedenken in Richtung einer als unzureichend wahrgenommenen Sicherheit und Systemzuverlässigkeit und machen die Gefahr datenschutzrechtlicher Übergriffe durch Produzenten und Betreiber zum gegenwärtigen Zeitpunkt für die geringe Akzeptanz dieser Technik verantwortlich - Aspekte, die einem Markterfolg im Privatkundensegment abträglich sind. Aus der Kritik der Befragten lässt sich institutionelle Verantwortung ableiten (Grunwald 2015, S. 682 ff.): Die Produzenten haben nicht nur für sichere, zuverlässige und resiliente Technik zu sorgen, sondern auch die verfassungsrechtlich garantierte informationelle Selbstbestimmung ihrer Kunden durch Einhaltung des Datenschutzes zu gewährleisten, die Politik hat die Sicherheit der Systeme und die Einhaltung des Datenschutzes zu überprüfen - auf diese Schlussfolgerungen weist auch eine aktuelle Studie des TÜV Rheinland hin (TÜV Rheinland 2018). Unsere Befunde geben Anlass zu der Vermutung, dass zumindest das Privatkundengeschäft in erheblichem Maße von der Glaubwürdigkeit und Leistungsfähigkeit der beiden Institutionen bei Datenschutz und Qualitätssicherung abhängt. Kritische Medienkommentare zu den jüngsten Unfällen mit voll autonomen Fahrzeugen mahnen, dass Feldversuche mit unausgereifter Technik dem Image autonomer Fahrzeuge und ihrer Produzenten nachhaltig schaden könnten (exemplarisch Pretzlaff 2016; Spiegel Online 2018 c; Doll 2018; Grunwald 2015, S. 680 ff.). ${ }^{5}$ Grunwald schreibt hierzu: „Wenn ein autonomes Fahrzeug wegen eines Softwarefehlers einen Unfall verursacht, ist das nicht akzeptabel. In einem solchen Fall ist anders als bei ,normalen ' Unfällen - eine erhebliche massenmediale Aufmerksamkeit zu erwarten. Insbesondere durch die

4 Exemplarisch sei an das Motto „Freude am Fahren“ von BMW (2018) erinnert, das der Automobilhersteller explizit mit „dynamischer Sportlichkeit“ verbindet. 5 Vorangegangene Unfälle, die von voll autonom fahrenden Fahrzeugen ausgingen, wurden schon Ende März 2018 von Tagesschau.de mit der Headline „Autonomes Fahren: Die Angst vor dem Autopiloten“ kommentiert (Tagesschau. de 2018 a). Am 24. Mai 2018 berichtete die Tagesschau über den Untersuchungsbericht zum Uber-Unfall in Arizona, am 30. Mai 2018 über die Kollision eines voll autonom fahrenden Tesla mit einem Polizeifahrzeug in Kalifornien (Tagesschau 2018 b und 2018 c). Eine Reihe von Kommentaren auf die Meldungen und das darin berichtete Technikversagen lassen erahnen, mit welch negativen Wirkungen durch derartige Unfälle auf die Technikakzeptanz gerechnet werden muss (Tagesschau.de $2018 \mathrm{~d}$ und $2018 \mathrm{e}$ ). 
Macht der Bilder und deren massenmediale Verbreitung können derartige Vorfälle eine erhebliche Wirkung [...] haben." (Grunwald 2015, S. 670)

Betrachten wir das zweite nachhaltigkeitsrelevante Kriterium: den in Aussicht gestellten verbesserten Zugang zu Mobilität und sozialer Teilhabe für Personen ohne Fahrerlaubnis, etwa durch Mobility-on-Demand-Angebote. Doch wie sehen das die Betroffenen selbst? In unserem Sample, das für die über 16-jährige deutschsprachige Wohnbevölkerung Repräsentativität beansprucht, haben wir untersucht, ob Personen, die zum Befragungszeitpunkt selbst keine Fahrerlaubnis hatten, Personen, die über 80 Jahre alt waren oder ihren Gesundheitszustand als eher schlecht oder schlecht einschätzen, durch eine überdurchschnittlich positive Haltung gegenüber dem voll autonomen Fahren auffallen. Keine der in Abbildung 1 aufgeführten Eigenschaften des autonomen Fahrens ist in nennenswerter Weise mit dem Besitz einer Fahrerlaubnis, hohem Alter oder schlechtem Gesundheitszustand assoziiert. ${ }^{6}$ Offenbar wird von diesem prädisponierten Personenkreis das voll autonome Fahren nicht in besonderer Weise als willkommenes Mittel zur Verbesserung von Mobilität oder sozialer Teilhabe wahrgenommen, ein Argument, das sich nicht zugunsten der sozialen Nachhaltigkeit dieser Technik verwenden lässt.

Die ökologische Dimension der Nachhaltigkeit kann erst beurteilt werden, wenn die Technologie eingeführt ist. Die von Fraedrich et al. (2017, S. 21 ff.) angestellten Überlegungen verdeutlichen, dass das vollautonome Fahren aus ökologischer Perspektive zu gegenläufigen Wirkungen führen kann, deren Eintreten von verbrauchsarmen Fahrzeugen ein Guthaben für nicht-nachhaltige Fahrten gesammelt zu haben. Dennoch möchten wir die referierten Daten durch den Befund ergänzen, dass 57,0\% aller Befragten angeben, bei adäquatem Angebot, umweltfreundliche Verkehrsmittel bei der Verkehrsplanung zu bevorzugen, nur $22,8 \%$ lehnen das ab. Knapp zwei Drittel der von uns Befragten $(63,1 \%)$ geben an, im Alltag auf die Nutzung eines Pkw angewiesen zu sein. Von diesen äußern 47,4\% die verbale Absicht, bei einem guten ÖPNV-Angebot auf das Auto verzichten zu wollen. Es besteht keinerlei Zusammenhang zwischen dieser Einstellung und der Haltung zum autonomen Fahren.

\section{Resümee}

Will man Indizien diskutieren, die für oder gegen die Nachhaltigkeit des voll autonomen Fahrens sprechen, gilt es, zuvor einen kritischen Blick auf das Nachhaltigkeitskonzept zu werfen. Gestützt auf das Nachhaltigkeitsverständnis der Brundtland-Kommission (Hauff 1987) und die Definition nachhaltiger Mobilität von Grunwald und Kopfmüller (2012) plädieren die Autoren für ein dreidimensionales Nachhaltigkeitskonzept, in dem soziale, ökonomische und ökologische Nachhaltigkeit gleichwertig koexistieren. Die Frage nach der Nachhaltigkeit des voll autonomen Fahrens ist im Hinblick auf diese drei Dimensionen zu diskutieren. Mit der Marktreife des voll autonomen Fahrens wird hierzulande frühestens in einem Jahrzehnt gerechnet, weswegen es gegenwärtig nicht möglich ist, anhand von Umfragedaten be-

\section{Sicherheit und Systemzuverlässigkeit des autonomen Fahrens werden als unzureichend wahrgenommen. Dies erklärt die gegenwärtig geringe Akzeptanz der Technik.}

und Wirkmächtigkeit vorab nicht eingeschätzt werden können. Allzu oft erweisen sich selbst solche Innovationen, die explizit mit dem Versprechen einer Verringerung ökologischer Belastungen entwickelt und eingeführt wurden, im Rückblick aufgrund der bekannten Rebound-Effekte (exemplarisch Sonnberger und Deuschle 2014) als nicht nachhaltig, sondern verursachen additive Nutzungen oder ein moral licensing - also die individuelle Einschätzung, auf dem moralischen Konto durch die Nutzung

\footnotetext{
6 Bei den vorliegenden Daten wird die Stärke des statistischen Zusammenhangs jeweils mit dem Kontingenzkoeffizienten Cramers V ausgedrückt, der Werte zwischen 0 (,kein Zusammenhang ${ }^{\prime}$ ) und 1 (,perfekter Zusammenhang ${ }^{\prime}$ ) annehmen kann. In der Praxis gelten erst Werte ab 0,1 als substanziell und interpretationsfähig (Cleff 2015, S. 85). Bei allen der hier untersuchten rund zwei Dutzend Zusammenhängen blieb $V \leq 0,08$. Auch in einer einschlägigen Studie von Bertelsmann nimmt die Begeisterung für das autonome Fahren entlang der Altersgruppen streng monoton ab und das Misstrauen mit wachsendem Alter zu (Berteilsmann Stiftung 2017, S. 4).
}

lastbare Prognosen über soziale, ökonomische oder ökologische Nachhaltigkeitspotenziale abzugeben. Gleichwohl haben wir in unserem Beitrag einige Indizien zusammengetragen, die für die Einschätzung von Nachhaltigkeitspotenzialen relevant sind.

Für die soziale Nachhaltigkeit sprechen vor allem steigende Mobilitäts- und Teilhabechancen von Personen ohne Fahrerlaubnis, allen voran alte, kranke oder behinderte Menschen - Personengruppen, die sich in unserem Survey allerdings nicht durch ein überdurchschnittliches Interesse am voll autonomen Fahren auszeichnen. Möglicherweise wird ihre Zustimmung von den noch unbekannten Geschäftsmodellen, Preisen, Bedienbarkeit und gegebenenfalls der Kostenübernahmebereitschaft durch den Sozialstaat abhängen.

Die ökonomische Nachhaltigkeit wird sich am Markterfolg des voll autonomen Fahrens bemessen, und zwar in allererster Linie am Massenmarkt mit Privatkunden. Die Daten des TechnikRadars zeigen allerdings, dass es aufgrund einiger Ein- 
wände um die Akzeptabilität des voll autonomen Verfahrens in der deutschen Öffentlichkeit gegenwärtig nicht gut bestellt ist. Die Sicherheit, ein Bestandteil der Definition nachhaltiger Mobilität von Grunwald und Kopfmüller (2012), wird von den Befragten des TechnikRadars sehr kritisch eingeschätzt. Defizite werden vor allem in nicht erfüllten institutionellen Verantwortlichkeiten gesehen, wie etwa in der technischen Zuverlässigkeit, Verkehrssicherheit, Datenschutz und Datensicherheit. Darüber hinaus werden die Geschäftsmodelle und Preise erheblich über den Markterfolg entscheiden und nicht zuletzt, in wieweit es gelingt, die „Freude am Fahren“ glaubhaft mit neuen Inhalten $\mathrm{zu}$ füllen.

\section{Entscheidend für den Markterfolg \\ des autonomen Fahrens werden}

\section{Geschäftsmodelle, Preise und neue Inhalte für ,Freude am Fahren“.}

Die ökologischen Folgen des voll autonomen Fahrens sind unseres Erachtens am schwierigsten zu beurteilen, hängen sie doch nicht nur maßgeblich vom Markterfolg ab, sondern von einer Reihe derzeit ungewisser Fragen zur technischen Beschaffenheit (Antriebsform, Motorleistung) und zur planerischen und sozialen Einbettung (z. B. Privatbesitz vs. Sharing- bzw. Mobility-on-demand-Konzepte; Nutzung übergeordneter Steuerungsmöglichkeiten; Auswirkungen auf die ÖPNV-Nutzung). Es darf aber vermutet werden, dass mit steigenden Beteiligungschancen bisher vom motorisierten Individualverkehr ausgeschlossener Personen und einem großen Markterfolg des voll autonomen Fahrens das Verkehrsaufkommen insgesamt ansteigen und damit die sozialen und ökonomischen Dimensionen von Nachhaltigkeit in ein Spannungsverhältnis zu den Postulaten einer ökologischen Nachhaltigkeit geraten werden.

\section{Literatur}

Aral AG (Hg.) (2017): Aral Studie. Trends beim Autokauf 2017. Bochum: Aral Aktiengesellschaft. Online verfügbar unter www.aral.de/content/dam/aral/ Presse\%20Assets/pdfs-Broschueren/Aral-Studie-Trends-beim-Autokauf-2017. pdf, zuletzt geprüft am 11.06.2018.

Bertelsmann Stiftung (Hg.) (2017): Automatisiertes Fahren. Aktuelle Einstellungen in Deutschland. Gütersloh. Online verfügbar unter https://www.bertelsmannstiftung.de/fileadmin/files/BSt/Publikationen/GrauePublikationen/ Factsheet_LK_Automatisiertes-Fahren_Aktuelle-Einstellungen-inDeutschland_2017.pdf, zuletzt geprüft am 11.06.2018.

BMW (2018): Freude am Fahren hat einen Namen. Online verfügbar unter www.bmwgroup.com/de/marken/bmw.html, zuletzt geprüft am 11.06.2018.

Cacilo, Andrej et al. (2015): Hochautomatisiertes Fahren auf Autobahnen. Industriepolitische Schlussfolgerungen. Stuttgart: Fraunhofer Institut für Arbeitswirtschaft und Organisation.
Cleff, Thomas (2015): Deskriptive Statistik und moderne Datenanalyse. Eine computergestützte Einführung mit Excel, SPSS und STATA. Wiesbaden: Springer.

Dangschat, Jens (2017): Automatisierter Verkehr - was kommt auf uns zu? In: Zeitschrift für Politikwissenschaft 27 (4), S. 493-507.

Deloitte (Hg.) (2017): What's ahead for fully autonomous driving? Consumer opinions on advanced vehicle technology. Perspectives from Deloitte's global automotive consumer study. Online verfügbar unter www2.deloitte.com/ content/dam/Deloitte/de/Documents/manufacturing/us-manufacturingconsumer-opinions-on-advanced-vehicle-technology.pdf, zuletzt geprüft am 16.05.2018.

Deutsche Bank Research (Hg.) (2017): Das „digitale Auto“. Mehr Umsatz, mehr Konkurrenz, mehr Kooperation. Deutschland-Monitor Digitale Ökonomie und struktureller Wandel. Frankfurt a. M. Online verfügbar unter www.dbresearch. de/PROD/RPS_DE-PROD/PROD00000000000445411/Das_\%E2\%80\%9Edigitale_ Auto\%E2\%80\%9C\%3A_Mehr_Umsatz\%2C_mehr_Konkurrenz\%2C.pdf, zuletzt geprüft am 11.06.2018.

Die Welt (2018): Lizenz weg. Unfall mit Roboter-Auto hat Folgen für Uber. Online verfügbar unter www.welt.de/wirtschaft/article174937737/Autonomes-FahrenToedlicher-Uber-Crash-wird-Roboterautos-nicht-bremsen.html, zuletzt geprüft am 11.06.2018.

Ernst \& Young GmbH (Hg.) (2017): Autonomes Fahren in Deutschland. Ergebnisse einer Befragung von 1.000 Verbrauchern. Eschborn: Ernst \& Young GmbH. Online verfügbar unter www.ey.com/Publication/vwLUAssets/ey-autonomesfahren-in-deutschland/\$FILE/ey-autonomes-fahren-in-deutschland.pdf, zuletzt geprüft am 11.06.2018.

Fraedrich, Eva et al. (2017): Automatisiertes Fahren im Personen- und Güterverkehr. Auswirkungen auf den Modal-Split, das Verkehrssystem und die Siedlungsstrukturen. In: e-mobil BW GmbH - Landesagentur für Elektromobilität und Brennstoffzellentechnologie Baden-Württemberg ( $\mathrm{Hg}$.): Publikationen der e-mobil BW. Stuttgart. Online verfügbar unter www.e-mobilbw.de/files/e-mobil/content/DE/Publikationen/PDF/PDF_2017/ Studie_AutomatisiertesFahren.pdf, zuletzt geprüft am 11.06.2018.

Friedrich, Bernhard (2015): Verkehr. In: Markus Maurer, Christian Gerdes, Barbara Lenz und Hermann Winner (Hg.): Autonomes Fahren. Technische, rechtliche und gesellschaftliche Aspekte. Heidelberg: Springer, S. 309-311.

Funk, Jeffrey (2015): IT and sustainability. New strategies for reducing emissions and resource usage in transportation. In: Telecommunications Policy 39 (10), S. 861-874.

Gabler, Siegfried; Häder, Sabine; Lehnhoff, Iris; Maridian, Elisabeth (2012): Weighting for unequal inclusion probabilities and nonresponse in dual frame telephone surveys. In: Sabine Häder, Michael Häder und Mike Kühne (Hg.): Telephone surveys in Europe. Research and practice. Heidelberg: Springer, S. 147-167.

Grunwald, Armin (2015): Gesellschaftliche Risikokonstellationen für autonomes Fahren. Analyse, Einordnung und Bewertung. In: Markus Maurer, Christian Gerdes, Barbara Lenz und Hermann Winner (Hg.): Autonomes Fahren. Technische, rechtliche und gesellschaftliche Aspekte. Heidelberg: Springer, S. 661-685.

Grunwald, Armin; Kopfmüller, Jürgen (2012): Nachhaltigkeit. Frankfurt a. M.: Campus.

Hampel, Jürgen; Zwick, Michael (2016): Wahrnehmung, Bewertung und Akzeptabilität von Technik. In: TATuP - Zeitschrift für Technikfolgenabschätzung in Theorie und Praxis, 25 (1), S. 24-38. 
Hauff, Volker (Hg.) (1987): Unsere gemeinsame Zukunft. Der Brundtland-Bericht der Weltkommission für Umwelt und Entwicklung. Greven: Eggenkamp.

Kopfmüller, Jürgen et al. (2001): Nachhaltige Entwicklung integrativ betrachtet. Konstitutive Elemente, Regeln, Indikatoren. Berlin: edition sigma.

Minx, Eckard; Dietrich, Rainer (2015): Autonomes Fahren. Wo wir heute stehen und was noch zu tun ist. Ladenburg: Piper.

Renn, Ortwin; Deuschle, Jürgen; Jäger, Alexander; Weimer-Jehle, Wolfgang (2007): Leitbild Nachhaltigkeit. Eine normativ-funktionale Konzeption und ihre Umsetzung. Wiesbaden: VS-Verlag.

Schreurs, Miranda; Steuwer, Sybil D. (2015): Autonomous driving. Political, legal, social and sustainability dimensions. In: Markus Maurer, Christian Gerdes, Barbara Lenz und Hermann Winner (Hg.): Autonomes Fahren. Technische, rechtliche und gesellschaftliche Aspekte. Heidelberg: Springer, S. 151-173.

Sonnberger, Marco; Deuschle, Jürgen (2014): Maßnahmen zur Eindämmung von Rebound-Effekten im Wohn- und Mobilitätsbereich. Ergebnisse aus zwei Expertenworkshops. In: Stuttgarter Beiträge zur Risiko- und Nachhaltigkeitsforschung 31. Stuttgart: Universität Stuttgart.

Spiegel Online (2016): Tödlicher Unfall in USA. Wie gefährlich sind selbstfahrende Autos? Online verfügbar unter www.spiegel.de/auto/aktuell/tesla-diewichtigsten-fakten-zum-toedlichen-unfall-in-den-usa-a-1100803.html, zuletzt geprüft am 11.06.2018.

Spiegel Online (2018 a): USA. Tödlicher Unfall mit selbstfahrendem Uber-Auto. Online verfügbar unter www.spiegel.de/wirtschaft/unternehmen/ubertoedlicher-unfall-mit-selbstfahrendem-auto-a-1198884.html, zuletzt geprüft am 11.06.2018.

Spiegel Online (2018 b): Nach tödlichem Zusammenprall. US-Behörde untersucht Unfall von Tesla-Fahrzeug. Online verfügbar unter www.spiegel.de/ auto/aktuell/us-behoerde-untersucht-unfall-von-tesla-fahrzeug-aktiefaellt-a-1200209.html, zuletzt geprüft am 11.06.2018.

Spiegel Online (2018 c): Tödlicher Uber-Unfall. Fürchtet euch ruhig. Online verfügbar unter www.spiegel.de/auto/aktuell/uber-unfall-die-furcht-vorder-kuenstlichen-intelligenz-am-steuer-a-1199188.html, zuletzt geprüft am 11.06.2018.

Stuttgarter Nachrichten (2016): Kommentar zum Tesla-Unfall. Die Risiken der Roboter-Autos. Online verfügbar unter www.stuttgarter-nachrichten.de/ inhalt.kommentar-zum-tesla-unfall-die-risiken-der-roboterautos.36129819a9bf-4e7b-aa25-d1bd0bedf42e.html, zuletzt geprüft am 11.06.2018.

Tagesschau (2018 a): Autonomes Fahren: Die Angst vor dem Autopiloten. Online verfügbar unter www.tagesschau.de/wirtschaft/autonomes-fahren-109.html, zuletzt geprüft am 11.06.2018.

Tagesschau (2018 b): Bericht zu tödlichem Unfall. Software erkannte Fußgängerin zu spät. Online verfügbar unter www.tagesschau.de/wirtschaft/uberunfall-101.html, zuletzt geprüft am 11.06.2018.

Tagesschau (2018 c): Unfall mit Autopilot: Tesla kracht in Polizeiauto. Online verfügbar unter www.tagesschau.de/ausland/tesla-unfall-113.html, zuletzt geprüft am 11.06.2018.

Tagesschau (2018 d): Ihre Meinung zu: Tödlicher Unfall. Uber-Software erkannte Fußgängerin zu spät. Online verfügbar unter https://meta.tagesschau.de/ id/134499/toedlicher-unfall-uber-software-erkannte-fussgaengerin-zu-spaet, zuletzt geprüft am 11.06.2018.

Tagesschau (2018 e): Ihre Meinung zu: Unfall mit Autopilot. Tesla kracht in Polizeiauto. Online verfügbar unter https://meta.tagesschau.de/id/134671/ unfall-mit-autopilot-tesla-kracht-in-polizeiauto, zuletzt geprüft am 11. 06.2018.
Thomopoulos, Nikolas; Givoni, Moshe (2015): The autonomous car. A blessing or a curse for the future of low carbon mobility? An exploration of likely vs. desirable outcomes. In: European Journal of Futures Research 3 (1), S. 1-14.

TÜV Rheinland (Hg.) (2018): TÜV-Rheinland-Studie zur Sicherheit autonomer Fahrzeuge. Fahrer wollen jederzeit eingreifen können. Repräsentative Befragung von Autofahrern in Deutschland, USA und China (Pressemitteilung). Online verfügbar unter https://www.tuv.com/de/deutschland/ueber_uns/ presse/meldungen/newspdfde_358792.jsp, zuletzt geprüft am 11.06.2018.

Umweltbundesamt (Hg.) (2005): Qualitätsziele und Indikatoren für eine nachhaltige Mobilität. Dessau: Umweltbundesamt.

Verband der Automobilindustrie (Hg.) (2015): Automatisierung. Von Fahrerassistenzsystemen zum automatisierten Fahren. Berlin: VDA.

Vereinte Nationen (1992): Rio-Erklärung über Umwelt und Entwicklung, Rio de Janeiro. Online verfügbar unter www.un.org/depts/german/conf/agenda21/ rio.pdf, zuletzt geprüft am 27.4.2018.

Wadud, Zia; MacKenzie, Don; Leiby, Paul (2016): Help or hindrance? The travel, energy and carbon impacts of highly automated vehicles. In: Transportation Research 86, S. 1-18.

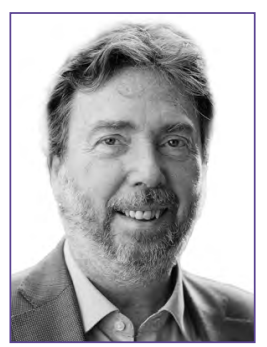

\section{DR. JÜRGEN HAMPEL}

ist akademischer Mitarbeiter am Lehrstuhl für Technik- und Umweltsoziologie der Universität Stuttgart und Mitglied des Zentrums für Interdisziplinäre Risiko- und Innovationsforschung der Universität Stuttgart. Seine Arbeitsschwerpunkte umfassen die soziologische Technik-, Risiko- und Umweltforschung, insbesondere die sozialwissenschaftliche Analyse gesellschaftlicher Reaktionen auf neue Technologien und dabei auftretender Konflikte.

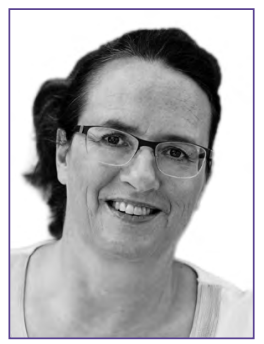

\section{PROF. DR. CORDULA KROPP}

ist Inhaberin des Lehrstuhls für Technik- und Umweltsoziologie und Direktorin des Zentrums für interdisziplinäre Risiko- und Innovationsforschung der Universität Stuttgart. Zu ihren Arbeitsgebieten zählen die Umwelt-, Technik- und Risikosoziologie, Forschung zum Wandel der Infrastruktursysteme in modernen Gesellschaften sowie Transformationsprozesse und soziale Innovation.

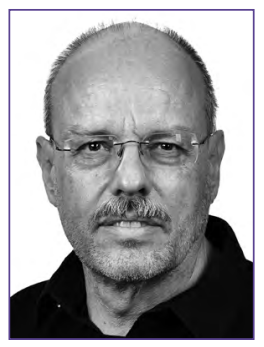

\section{DR. MICHAEL M.ZWICK}

arbeitet als Technik- und Umweltsoziologe am Institut für Sozialwissenschaften der Universität Stuttgart. Zu seinen Arbeitsschwerpunkten zählen neben der Umwelt- und Risikoforschung die Wahrnehmung des technischen Wandels in der Öffentlichkeit sowie qualitative und quantitative Methoden der empirischen Sozialforschung. 